Research Article

\title{
An Adaptive Trend Index-Driven Remaining Useful Life Prediction Method for Renewable Energy Vehicle Reducers
}

\author{
Feng Chen $\mathbb{D},{ }^{1,2}$ Weilin Li $\mathbb{C}^{2},{ }^{2}$ Wenxiang Weng, ${ }^{2}$ Xiaoyv Sheng, ${ }^{1}$ Binghai Lyu, \\ Qinghua Yang $\mathbb{D}^{1}{ }^{1}$ and Libin Zhang ${ }^{1}$
}

${ }^{1}$ College of Mechanical Engineering, Zhejiang University of Technology, Hangzhou 310023, China

${ }^{2}$ Zhejiang Fangyuan Test Group Co., Ltd., Hangzhou 310018, China

Correspondence should be addressed to Qinghua Yang; robot@zjut.edu.cn

Received 14 June 2021; Revised 24 July 2021; Accepted 30 July 2021; Published 8 October 2021

Academic Editor: Liang Guo

Copyright (c) 2021 Feng Chen et al. This is an open access article distributed under the Creative Commons Attribution License, which permits unrestricted use, distribution, and reproduction in any medium, provided the original work is properly cited.

Renewable energy vehicle reducers are now being developed towards achieving high-speeds, high-torque, and high-integration and intelligent trends. Its performance also determines the operation state and reliability of vehicles. Therefore, it is necessary to conduct the online condition assessment and remaining useful life predictions for renewable energy vehicle reducers. In those methods, the trend index construction is one of the most crucial steps. Hence, an adaptive trend index-driven remaining useful life prediction method is proposed to conduct condition assessment and prediction of renewable energy vehicle reducers. Firstly, an adaptive trend index is constructed, where the difference of the Fourier amplitude spectrum between the initial state and the current state is calculated to present the health trend index. Secondly, the reducer's performance degradation model is built. In order to conduct remaining useful life prediction, the particle filtering is used to update the parameters of the reducer's performance degradation model with the constructed adaptive trend index. In order to verify the effectiveness of the proposed method, an accelerated life test is conducted on a three-motor test bed to achieve the life-cycle data of reducers. The proposed method is verified with the obtained data and compared with the commonly used ARIMA model. The test results show that the proposed method achieves better results than the traditional methods. It means that the proposed method is a potential one for the real-time monitoring of the health state of renewable energy vehicle reducers.

\section{Introduction}

Renewable energy vehicles are being developed with the aim of advancing their intelligence, high performance, and shorten development cycle. To conform with this developmental trend, reducers, as one of the key elements in an automotive transmission system, are also being gradually developed towards having a higher speed, torque, and integration. The state of the reducer decides the power, comfort, and reliability of vehicles. It is therefore necessary to conduct several life-cycle tests on the reducer during its developmental stage. However, such tests are time-consuming and costly, and the reducers' failure time is uncertain. Therefore, in order to timely make managerial decisions on the vehicle's health, an online condition assessment method should be first carried out to determine the fatigue life of the renewable energy vehicle's reducers and its remaining useful life (RUL) prediction [1-3].

The RUL prediction consists of a quantitative assessment of the remaining lifespan of the existing service components. The RUL of a vehicle's reducer is mainly predicted using data-driven methods and physical model-based methods [4-6]. For the accurate prediction, the physical model requires specified reducer-related system and theory knowledge. The data-driven model mainly predicts the remaining useful life according to the signal processing, statistical, artificial intelligence theory, and methods, instead of relying on engineering practice principles. The data-driven RUL prediction method thus already becomes the core one of equipment prediction and maintenance.

Currently, the shallow neural network [7], support vector machine [8], deep learning $[9,10]$, and other algorithms have 
already been applied for reducer-related data-driven RUL predictions. Pan et al. [11] proposed a performance degradation assessment method based on complete ensemble empirical mode decomposition with adaptive noise and kernel principal component analysis. In the proposed method, an extreme learning machine is used to denoise and fuse the vibration signals and predict the RUL of the gearbox. Chen et al. [12] proposed a life prediction method based on the relative features and multielement support vector machine. A multielement support vector machine considers the interaction among all variables and can obtain information from small samples. According to the test, this method has been proven to accurately predict the remaining useful life of a bearing. Wang et al. [13] proposed a recurrent convolutional neural network-based RUL method. In the proposed method, the monitoring data were directly input into the recurrent convolution neural network to conduct RUL prediction of machines. Guo et al. [14] built a recurrent neural network-based health indicator construction method to conduct RUL prediction of bearings. In the proposed method, lots of life-cycle bearing data were used to train and test the proposed model. The abovementioned intelligent model-based RUL prediction method usually requires lots of life-cycle data for the model training, but it is often difficult to achieve large amounts of life-cycle data in industrial application. This is especially true in the renewable energy vehicle reducer field as we are still in the initial stages of acquisition for the monitoring of data. Due to this lack of data, it would be difficult to satisfy the requirements for predicting the RUL of renewable energy vehicle reducers using the abovestated intelligent model.

An alternative has recently been proposed by many scholars which is based on the random filtering theory. Additionally, a random degradation model is usually been established to predict the RUL and guarantee the reliability of reducers [15-17]. Teng et al. [18] extracted the vibration signal features of full-cycle data on a gearbox and predicted the RUL using the Hidden Markov Model. Li et al. [19] considered the competing risk model within the semiMarkov decision process framework to make maintenance decisions on the early failure detection of gear systems. During the modelling process, the parameter estimation on the internal state of machines based on the monitoring value requires a consideration of the impact of noise. To accurately estimate the remaining life of machines, particle filtering and the state space equation can be used. Sun et al. [20] conducted state space modelling for the remaining life degradation model of a gearbox and proposed a life prediction method based on particle filtering. Cheng et al. [21] came up with an improved particle filtering method and designed an adaptive neural network fuzzy inference system to predict the RUL of bearings in the wind turbine gearbox with the fault index extracted from the monitoring data.

The most crucial step of RUL prediction methods based on random filter theory is the construction of appropriate trend indexes [22-24]. Currently, most of trend indexes are mainly constructed based on the manual feature extraction method. Li et al. [25] proposed a trend index construction method with the Kullback-Leibler distance under $\alpha$ stable distribution. The effectiveness of the proposed method was verified by the bearing test data. Qiu et al. [26] put forward a trend index construction method from frequency spectrum structural information and used it to verify the life predictions of rolling bearings. Jin et al. [27] extracted the root mean square error as the trend index to represent the state of the bearing's health and provide support for RUL prediction. Duong et al. [28] extracted the discrete wavelet packet transform features of the bearing vibration signals and then smoothed out the most characteristics features to construct the trend index. The abovementioned trend index construction method is based on the manual feature extraction methods, and they are used to extract the change of the absolute value of monitoring signals, which is greatly impacted by the operating condition. In order to eliminate the impact of such factors, the relative-change features are needed.

This paper puts forward an adaptive trend index-driven method for the life prediction of renewable energy vehicle reducers. The method mainly includes three steps: data acquisition, trend index construction, and RUL prediction. In the data acquisition stage, the vibration signals from the reducers during the life-cycle process are obtained through the acceleration sensors, and the data are preprocessed. In the trend index construction stage, a self-updating method is proposed to calculate the variation trends in the monitoring data of the reducers during different performance states, relative to the initial state. In the RUL prediction stage, the parameters for the updating of the performance degradation model are based on particle filtering. An accelerated life test is conducted for the renewable energy vehicle reducers in which the casing was reduced through a comprehensive performance three-motor test bed to obtain the life-cycle data of the reducers. The data are then applied to verify the effectiveness of this method.

The main contributions are mainly summarized as follows. (1) A new adaptive trend index construction method is proposed. The relative difference of the Fourier amplitude spectrum between the initial state and the current state is calculated as a new trend index to present the health condition. (2) A single-exponential model is built to conduct RUL prediction of renewable energy vehicle reducers. The model parameters are updated through particle filtering with the constructed trend index. (3) The accelerated life test of renewable energy vehicle reducers is conducted to acquire the full-cycle data. Those data are important for verifying the effectiveness of the proposed method.

\section{The Proposed Method}

The reducer's life prediction is mainly implemented in three steps: data acquisition and preprocessing, trend index construction, and RUL prediction. In the data acquisition and preprocessing stage, the reducer's monitoring data are obtained through order sampling. In the trend index construction stage, using the frequency spectrum of the monitoring data in the initial stage as the reference, the difference between the frequency amplitude of the monitoring data and the reference frequency spectrum is calculated. In the RUL prediction stage, performance degradation curve parameters are fitted with the trend index for RUL prediction. This process is shown in Figure 1. 


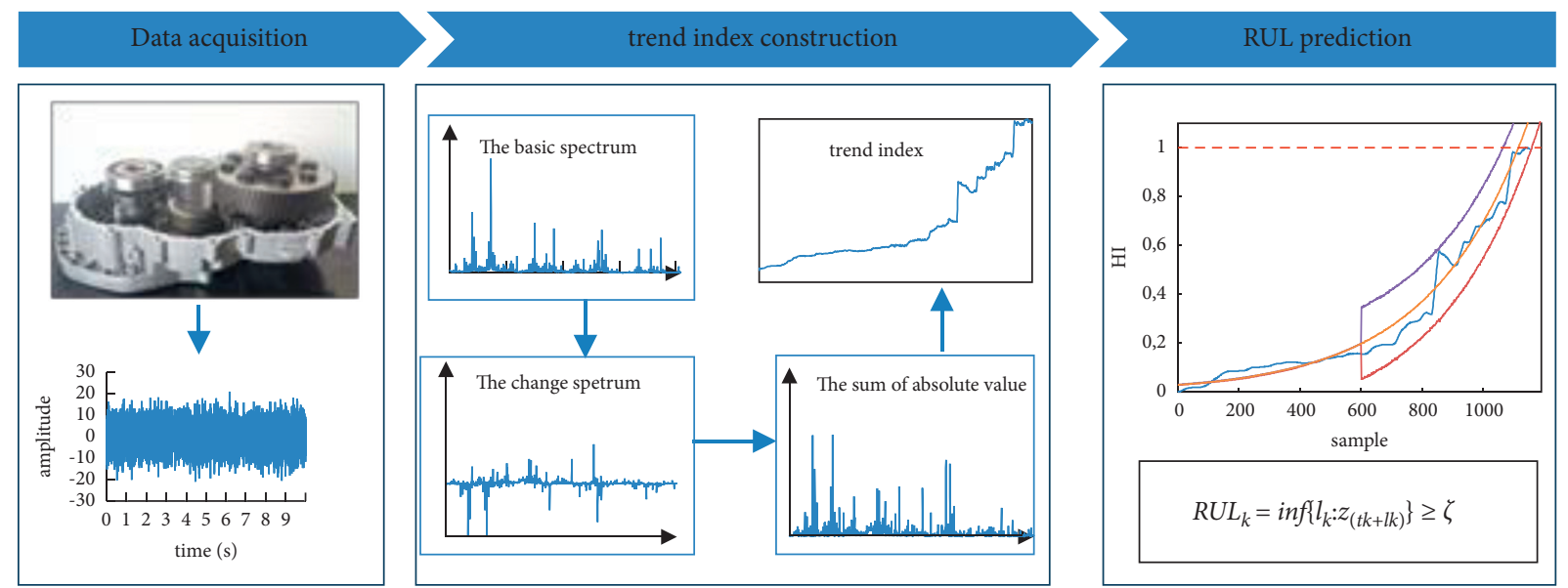

Figure 1: Data-driven online condition assessment and life prediction of renewable energy vehicle reducers.

2.1. Data Acquisition and Preprocessing. A renewable energy vehicle reducer is installed on the test bed. The test bed is operated with the specified working conditions for testing. The monitoring data from the reducer's vibration sensor are obtained through high frequency sampling software. To accurately distinguish between different working conditions and find the implicit feature information, the data are classified and collected. To eliminate the rotating speed's impact on the frequency domain of the fault features, in addition to the vibration signals, the rotating speed signals are also collected during the sampling process for order sampling.

Fourier transform is conducted for the collected time domain data in order to obtain the frequency domain data. The frequency domain data could be directly collected according to the sampling device's functions and used as the basic input data of the subsequent health trend index construction.

2.2. Trend Index Construction. This paper puts forward a self-updating trend index construction method to calculate the variation trend in the monitoring data of the reducer in the different performance states, relative to the initial state. The proposed method covers three steps, including reference learning, offset tolerance calculation, and trend index construction.

In the stage of standard learning, the average frequency spectrum of the signals collected $n$ times is calculated. Then, the mean value of the frequency spectrum is analyzed as the reference value as follows:

$$
\bar{x}_{b}=\frac{\sum_{i=1}^{n} X_{i}}{n}
$$

where $X_{i}$ is the frequency spectrum of signals collected for the $i^{\text {th }}$ time, $\bar{x}_{b}$ is a reference value, and $n$ is the number of training sets in the learning stage.

In the offset tolerance calculation stage, the frequency spectrum of the signals collected for five continuous times is calculated. Following this, the offset tolerance between the frequency spectrum of the signals collected each time and the reference frequency spectrum is analyzed. The specific steps are as follows:

$$
\begin{aligned}
S_{1}= & \frac{\sum_{i=n+1}^{i=n+5} X_{i}}{5}-\bar{x}_{b}, \\
S_{2}= & \frac{\sum_{i=n+2}^{i=n+6} X_{i}}{5}-\bar{x}_{b}, \\
& \ldots \\
S_{K}= & \frac{\sum_{i=n+N-4}^{i=N} X_{i}}{5}-\bar{x}_{b}, \\
T I_{t}= & \sum S_{i}, \quad i=1,2,3, \ldots, K,
\end{aligned}
$$

where $N$ is the number of sample data sets, $T I_{t}$ is the trend index value at the current moment, and $S_{1} \sim S_{K}$ is the respective offset tolerance from the first to the $K^{\text {th }}$ spectral line.

Finally, in the trend index construction stage, the trend index at each moment is spliced into the constructed trend index of the reducer as follows:

$$
T I=\left\{T I_{t}\right\}_{t=1: K}
$$

where $K$ is the length of $T I$ sequence, and $K=N-n-4$.

In conclusion, an autocorrelated trend index construction method is built. The trend index of the current state is compared with its initial state, which can be used to characterize the development process of the reducer performance state. With the mean value of the feature in the initial frequency dataset with domain signals as a reference, the mean value is calculated one time once five data sets had been collected. This is done to work out the deviation of the two and so the variation spectrum is generated. Next, the trend index of a point is formed by adding up the variation spectrum, while the trend index curve which changes with the timeline is formed by variation spectrums, namely, the reducer's trend index (TI). Please refer to the specific process as follows (Algorithm 1):

2.3. RUL Prediction. According to the constructed trend index, a good trend of the indexes' time domain features could be observed. In order to conduct a real-time 


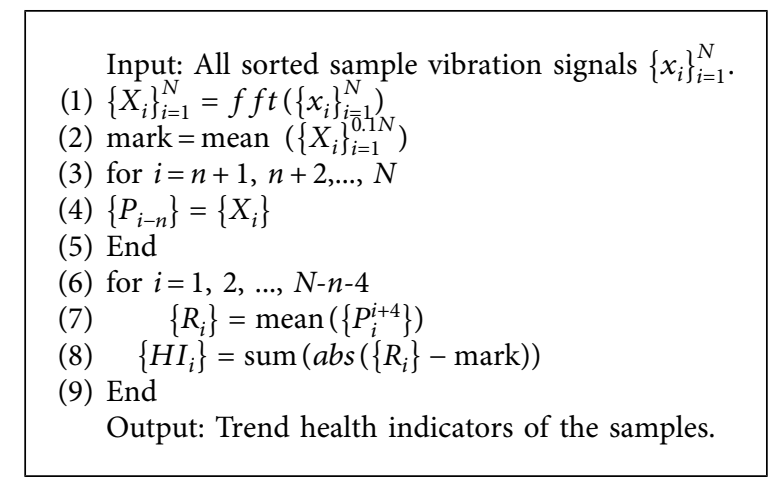

Algorithm 1: The process of constructing the trend index (TI).

evaluation of the health state of the reducers and the RUL prediction, the particle filtering algorithm and the mathematical model that describes the system degradation process and constructs the trend index are used to conduct a mathematical model linked with the RUL prediction of the reducers.

Bayesian theory can solve this type of state estimation problem, as well as conduct a recursive estimation of the device's future state value by taking the process monitoring data (usually the sequential observation value) as the prior information. The sequential Monte Carlo method, also known as the particle filtering algorithm, is used to estimate the system's state in a nonlinear and non-Gaussian condition. Generally, the system's dynamic variation can be indicated by the state transition equation (4) and observation equation (5).

$$
\begin{gathered}
x_{k}=f_{k}\left(x_{k-1}, v_{k-1}\right), \\
z_{k}=h_{k}\left(x_{k}, n_{k}\right),
\end{gathered}
$$

where $x_{k}$ is the system state, $z_{k}$ is the system observation value, $f_{k}$ is the state transfer function, $h_{k}$ is the observation function, $V_{k-1}$ is the process noise, and $n_{k}$ is the observation noise.

The probability distribution of the system state $x_{k}$ in the current moment is inferred by the existing observation data. The process includes two steps: prediction and update. The particle filter is a nonparameter implementation of the Bayesian filter and is usually used to estimate a dynamic system state. The key to the particle filter's effectiveness lies in its use of a set of assumptions (namely, particles) to indicate posterior probability, with each assumption standing for a possible state of the system. The state assumption is represented by a set $S$ with $N$ weighted random samples:

$$
S=\left\{\left(s^{[i]}, \omega^{[i]} \mid i=1,2, \ldots, N\right)\right\},
$$

where $s^{[i]}$ is the state vector of the $i^{\text {th }}$ sample and $\omega^{[i]}$ is the weight of the $i^{\text {th }}$ sample. The weight is not 0 , and the sum of all the weights is 1 . The sample set represents the following distribution, namely, the posterior probability density of the system's states:

$$
p(x)=\sum_{i=1}^{N} \omega_{k}^{i} \cdot \delta_{s[i]}(x),
$$

where $\delta_{s[i]}$ is the Dirac function in state $s^{[i]}$ of the $i^{\text {th }}$ sample and $\omega_{i}$ is the normalized weight of the $k^{t h}$ particle. The update process can be simplified as the following equation:

$$
\begin{aligned}
& \omega_{k}^{i}=\omega_{k-1}^{i} \frac{p\left(z_{k} \mid x_{k}^{i}\right) p\left(x_{k}^{i} \mid x_{k-1}^{i}\right)}{q\left(x_{k}^{i} \mid x_{k-1}^{i}, z_{1: k}\right)}=\omega_{k-1}^{i} p\left(z_{k} \mid x_{k}^{i}\right), \\
&\left(\text { when } q\left(x_{k} \mid z_{1: k}\right)\right.\left.=p\left(x_{k} \mid x_{k-1}\right)\right) .
\end{aligned}
$$

Sample set $S$ can be used to approximate any distribution, while these samples are taken from this approximated distribution to specify such an approximation.

Through the trend index analysis of the experimental data, a single-exponential model is adopted as the degradation model. This results in a good fitting result, and the observation equation is as follows:

$$
z_{k}=a e^{b k}+n_{k}
$$

where $a$ and $b$ are model parameters, $k$ is the time point, and $n_{k}$ is the process noise.

After establishing the gearbox's degradation model, the reducer's RUL prediction could be conducted by combining the constructed trend index, as well as through the calculation of the particle filtering algorithm. The operation process is as follows:

(1) Determine the initial value of parameters a and $b$ through data fitting

(2) Divide some data for the initial training and division of the prediction interval

(3) Use the particle filtering method to assign values to the particle points in the prediction interval

(4) Construct a new trend index with the particle points

(5) Conduct an RUL prediction of the reducer with the value of the new trend index

The RUL of the reducer at $t_{k}$ moment is calculated by

$$
R U L_{k}=\inf \left\{l_{k}: z_{\left(t_{k}+l_{k}\right)}\right\} \geq \zeta
$$


Input: All trend index (TI).

(1) For $i=t: 10: T$.

(2) Carry out the initial value fitting of the parameters through TI [1:t].

(3) while $\min \left(\left\{x_{k}^{z}\right\}_{z=1}^{Z}\right)<$ Thres

(4) $\quad k=k+1$

(5) Importance sampling

(6) Weight update $w_{k}^{i}=\omega_{k}^{i} / \sum_{i=1}^{N} \omega_{k}^{i}$

(7) Resampling

(8) State estimation $\widehat{x_{k}}=X_{(N / 2)}$

(9) End.

(10) Calculate the residual life according to formula (8).

(11) End.

Output: Prediction results distribution of residual life.

Algorithm 2: Remaining useful life prediction.

where $\inf \{\cdot\}$ is the lower limit of the variable and $l_{k}$ is the time spent from the $k^{\text {th }}$ time point to the failure of the reducer. The specific calculation process is as follows (Algorithm 2).

\section{Experiment and Results}

3.1. Experimental Platform. A three-motor test bed will be built for the test research in order to obtain the original actual degradation data of the corresponding reducer for the poststage data processing, modelling, and life prediction.

This test platform can be used to test the performance, fatigue life, and other items of the renewable energy vehicle's driving system (electrically driven power assembly, hybrid power assembly, gearbox assembly, and so on). The test platform's sketch and physical maps are shown in Figure 2 and 3 , respectively. The test platform's main structure consists of three motors. Among these, one is the main drive motor used for driving the test piece to work, and the other two are load motors used for simulating the load functions. Both drive and load motors can be switched in order to implement simulation tests for forward and reverse drive conditions. These three motors function as both motor and generator, while the whole platform's electrical control system employs the AC closed energy recovery system based on the DC bus. To meet the test requirements for different temperature conditions, the platform is equipped with test temperature control devices, such as a low temperature cooling device and an environment room. Additionally, the detail parameters of vibration signal acquisition are shown in Table 1.

3.2. Experiments. The two-stage reducer from a typical renewable energy vehicle is adopted as the test piece for the accelerated life test. The max speed reaches $12000 \mathrm{r} / \mathrm{min}$. Please refer to Table 2 for the main parameters of the test piece. It is installed on the test platform according to the vehicle's installation method, and a certain type and amount of lubricant is added as per the requirements. The vibration sensor is installed on the casing near the high-speed shaft as shown in Figure 4.

The test piece underwent both running-in and fatigue life tests according to the "QC/T 1022-2015 Technical specification for reduction gearbox of battery electric passenger cars"-a

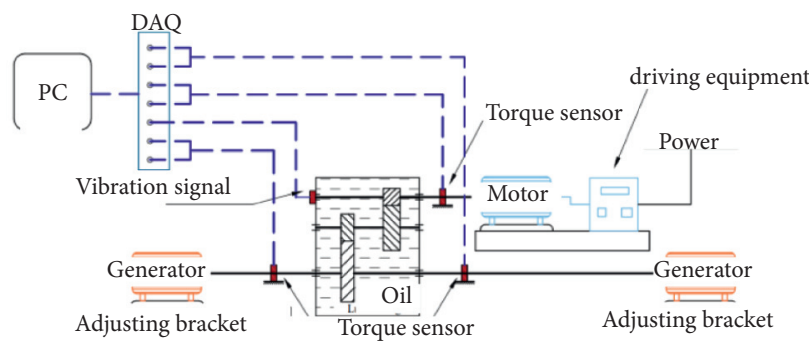

Figure 2: Principal chart of triaxial reducer test bed.

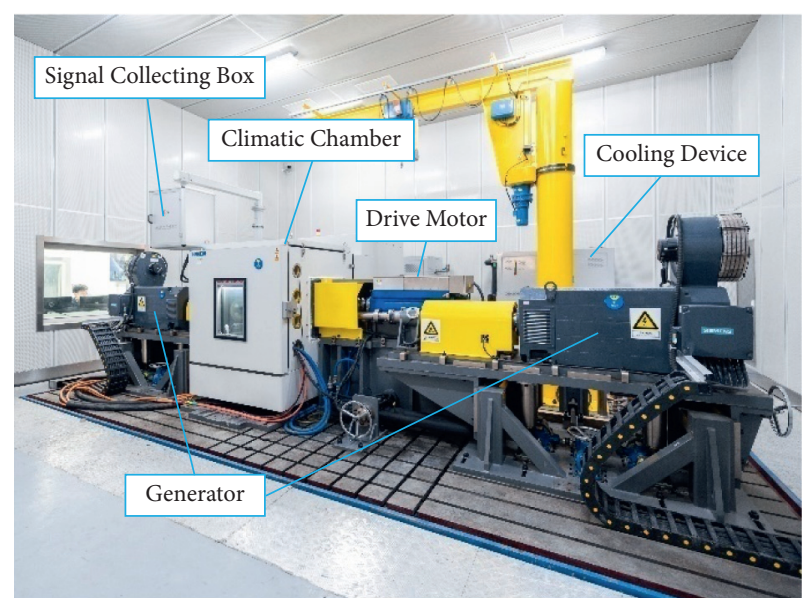

Figure 3: Triaxial reducer test bed.

TABle 1: Parameters of vibration center

\begin{tabular}{lc}
\hline Parameters & Value \\
\hline Measuring range $(\mathrm{r} / \mathrm{min})$ & $\pm 80 \mathrm{~g}$ \\
Sampling frequency & $24 \mathrm{KHz}$ \\
Number of axles & Single axis \\
Sensitivity & $26 \mathrm{mV} / \mathrm{g}$ \\
\hline
\end{tabular}

standard used in China's auto industry. Specifically, the vehicle ran for a total of 43 hours, including 33 hours in a high-torque forward driving condition, 4 hours in a high-torque reverse 
driving condition, followed by another 6 hours in a high-speed forward driving condition. This is equally divided into ten cycles for the test. Except for in instances of uncontrollable damage, the test is not stopped until all cycles have been completed. Two test pieces finish the life-cycle test. Of these, one finished the test normally with no serious failure, but the bearing is seriously worn; the other finished the test normally, and there is no serious failure, but the bearing is serious worn and gear pitting is also serious.

A data set is generated following the collection of 12288 pieces of data every 12 turns. Figure 5 displays the time domain curve of the data from test pieces 1 and 2 in different working conditions.

3.3. Trend Index Construction. Time-frequency transform was conducted for the collected signals. This method was divided into two parts that were seamlessly integrated: the learning phase and monitoring phase. From Figure 6, we observe the following: (a) the reference spectrum obtained from the mean value of a test piece's first ten pieces of data; (b) the mean value of the frequency spectrum of every adjacent five time points; (c) the allowance between frequency spectrum (b) and reference spectrum (a), where the variation spectrum can be formed no matter whether the deviation is positive or not; and (d) the absolute value of the variation spectrum (c). The trend index of a point can be formed by adding up the variation spectrum.

Several variation spectrums are obtained from the above-stated steps, while the trend index curve of samples 1 and 2 was obtained according to the time change. Based on the obtained two groups of the trend index, the window function is used for data mean filtering. The results are shown in Figure 7, where obvious monotony and tendency can be seen. The constructed trend index grows slightly at first and then sharply increases, which conforms to the gearbox degradation rules. It can be used to calculate the variation trend of the reducer's monitoring data in its different performance states relative to the initial state. The performance trend prediction is the foundation of life prediction, and its accuracy and predictability are closely related to the precision of the subsequent RUL prediction.

3.4. RUL Prediction. To determine the fitting function of the failure process, a feature analysis is conducted for the trend index obtained from the data processing. The precondition using the particle filtering algorithm fits the mathematical expression of the specified target's degradation process. In order to accomplish this, the form of the fitting function for the sample data's degradation process needs to be determined. The single-exponential and double-exponential models are the most widely used in particle filtering. During the fitting and analysis of the experimental data, the single-exponential model is eventually selected as the fitting function for the degradation process, and the fitting function for the trend index degradation is rewritten as follows:
TABLE 2: Parameters of some secondary reducers.

\begin{tabular}{lc}
\hline Parameter & Value \\
\hline Max speed (r/min) & 12000 \\
Max torque (N.M) & 210 \\
Max power (kW) & 90 \\
Gear type & Helical gear \\
\hline
\end{tabular}

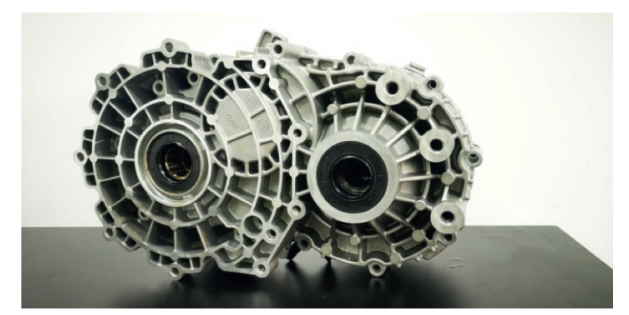

Figure 4: Secondary reducer.

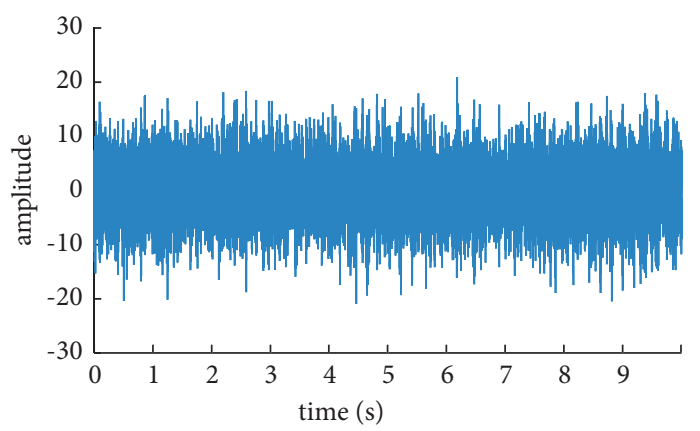

FIgURE 5: Dataset time domain curve.

$$
x_{k}=\exp \left(-b_{k} \Delta t\right) x_{k-1} .
$$

According to the determined fitting function, new samples were further divided for particle filtering. Since the sample set would greatly influence the final prediction results, the overall data distribution could be carried out in ideal conditions. If the overall distribution of the data was deemed to be the prior distribution, the fitting results could be employed to obtain relatively ideal experimental results. If the above-determined two groups of parameters are used directly, the particle filtering results are as shown in Figure 8. The initial parameters are shown in Table 3.

According to Figure 8, it can be clearly seen that the prediction results are basically consistent with the actual data. The actual data are within a 95\% confidence interval of the prediction results, and the deviation is slight at the failure threshold moment.

In the case of insufficient data, the life prediction could only be conducted on the basis of existing data. Therefore, it is necessary to reconduct the dataset division and fitting of the function curve. The most common modelling method is to extract a portion of the actual dataset as the training set. In the follow-up life prediction, two initial 


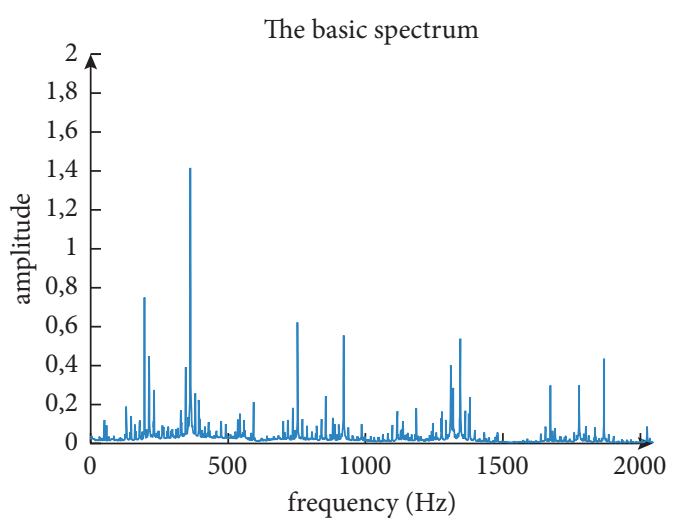

(a)

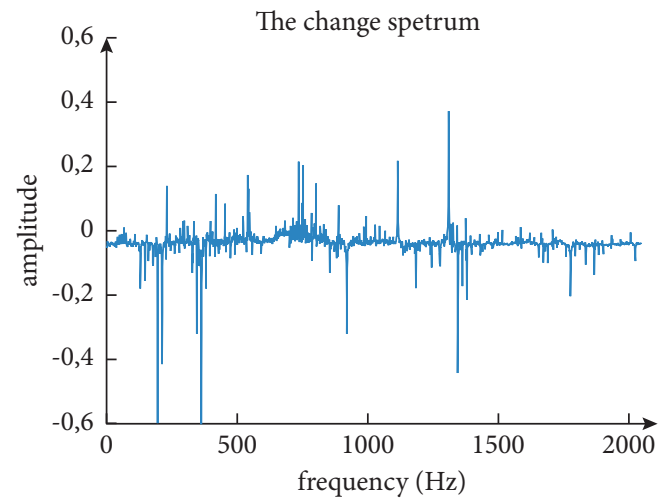

(c)

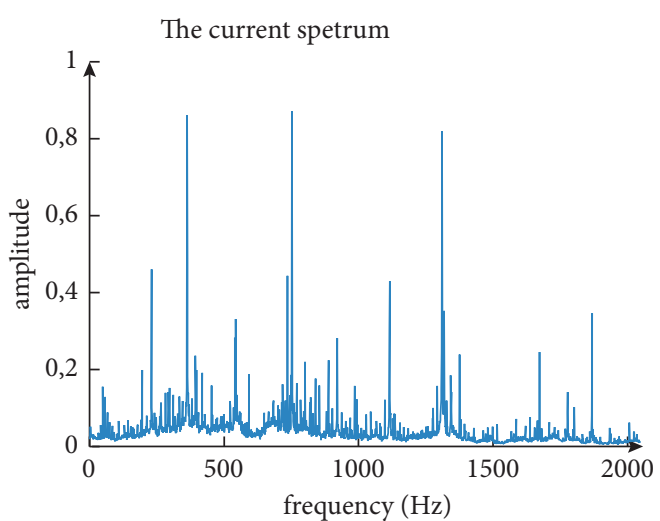

(b)

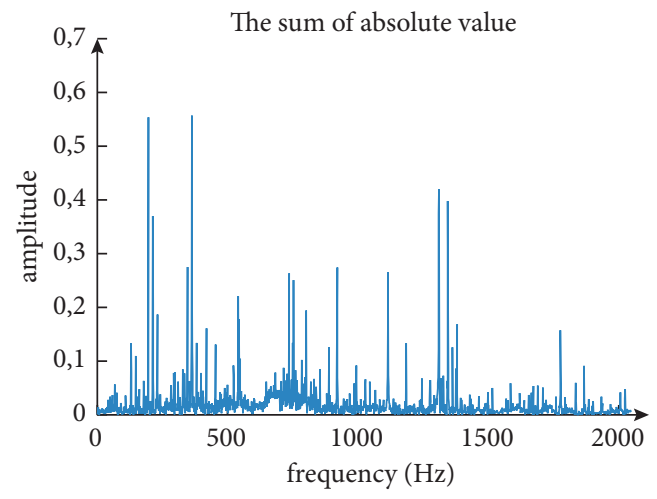

(d)

Figure 6: Frequency domain signal and offset tolerance calculation.
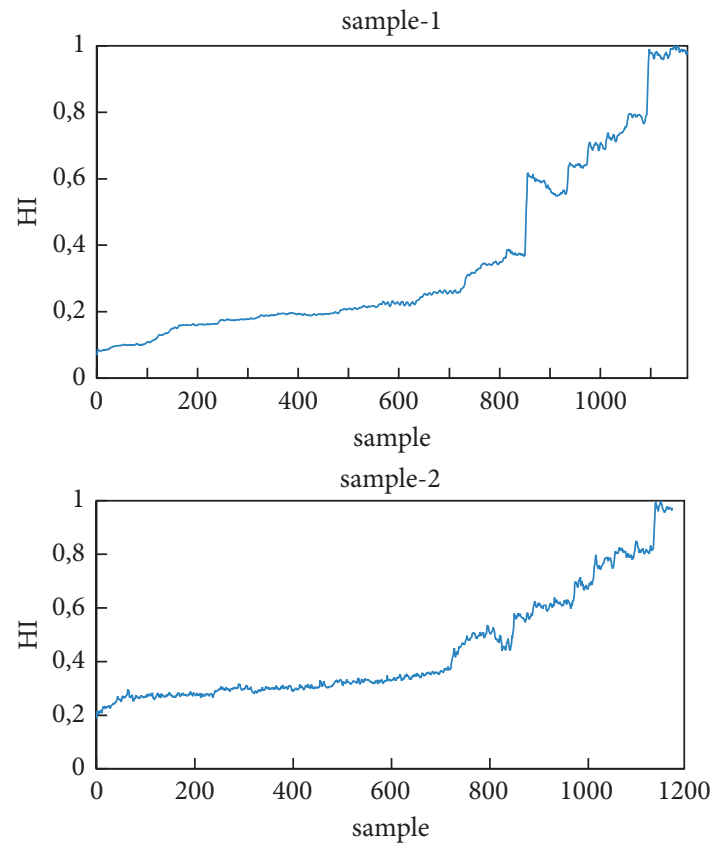

Figure 7: Trend index of two samples.

data samples are defined as 664 and 667, and ten samples are added in each training. The results are shown in Figure 9.
During the RUL prediction, the median particle distribution at each time point is taken as the predicted value at the current moment, while the RUL of the gearbox at this current moment is calculated according to the previously stated method. According to the previous fitting results and actual data, the failure threshold of the two samples is set to be 1 . The prediction results show that the prediction results of the two samples at the beginning are greater than the actual value, and that a great deviation occurs between the predicted RUL and actual RUL. With the passage of time, however, the observation data gradually increased, and the prediction results are shown to be closer to the actual RUL. Particle filtering increased with the samples, and its superiority is reflected.

To further verify the effectiveness of this method, the commonly used ARIMA model is applied for comparison.

ACF reflects the correlation between the values of the same sequence in different time series.

$$
\operatorname{ACF}(k)=\rho_{k}=\frac{\operatorname{Cov}\left(y_{t}, y_{t-k}\right)}{\operatorname{Var}\left(y_{t}\right)} .
$$

PACF is the relevance of $x(t-k)$ 's impact on $x(t)$ after eliminating the interference of the $k-1$ random variables, including $x(t-1), x(t-2), \ldots \ldots, x(t-k+1)$.

The formula definition of the p-order autoregressive process is as follows: 

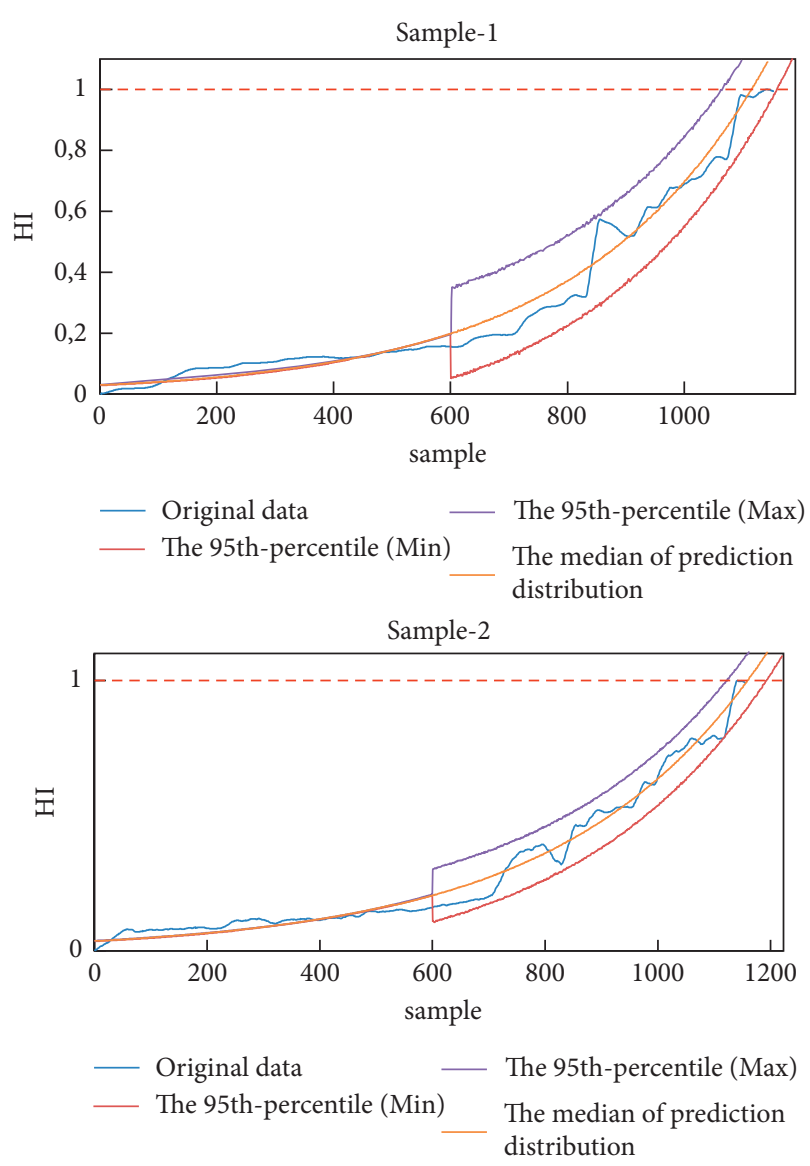

FIGURE 8: RUL prediction results.

TABLE 3: Initial parameters used in the prediction process.

\begin{tabular}{lcc}
\hline & Sample 1 & Sample 2 \\
\hline Total data length & 1154 & 1157 \\
Training set & $(1: 600)$ & $(1: 600)$ \\
Prediction set & $(601: 1154)$ & $(601: 1157)$ \\
Number of particles & 5000 & 5000 \\
Parameter b & $(0.003098,0.003171)$ & $(0.002819,0.002878)$ \\
Failure threshold & 1 & 1 \\
\hline
\end{tabular}

$$
y_{t}=\mu+\sum_{i=1}^{p} \gamma_{i} y_{t-i}+\varepsilon_{t},
$$

where $y_{t}$ is the current value, $\mu$ is a constant, $p$ is the order, $\gamma_{i}$ is the autocorrelation coefficient, and $\epsilon_{t}$ is the error.

The stationary time series is obtained after processing, and its ACF and PACF are worked out in order to determine the parameters of the ARIMA model, $p=1, d=1, q=1$, and $s=12$.

The difference method is applied for the model's prediction. According to Figure 10, based on the first 600 pieces of data in sample 1, the prediction results are to be greatly different of the actual data. Additionally, the predicted median point's trend within the distribution has no cross point with the failure threshold. Therefore, the method fails
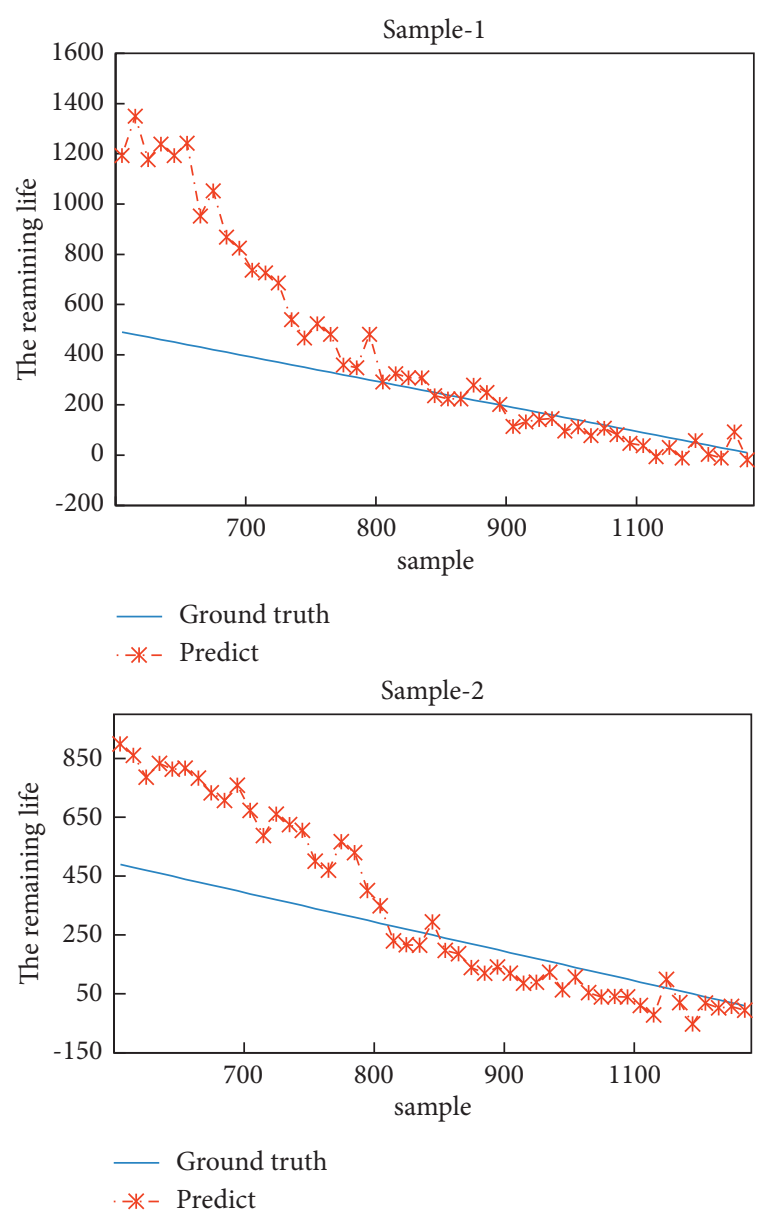

Figure 9: Results of several predictions.

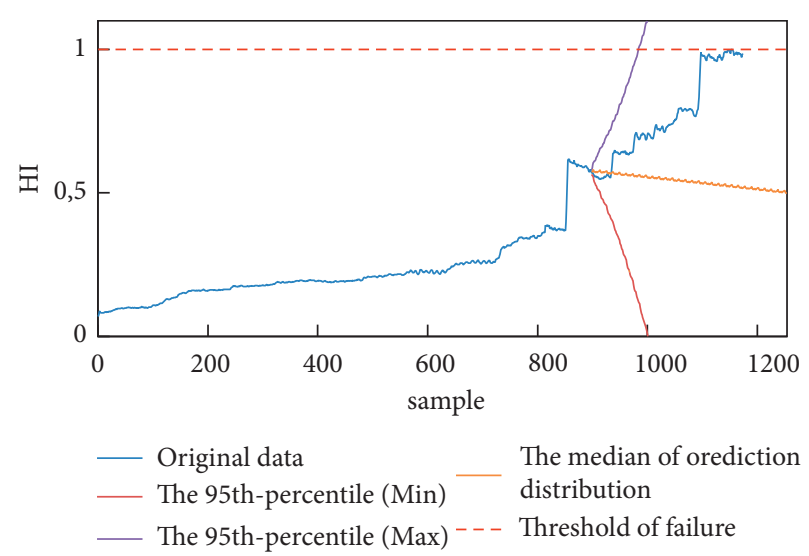

FIgURE 10: ARIMA-based prediction results for sample 1 (1).

to reasonably predict the great variation trend index and is clearly not as good as the method proposed in this paper. Consequently, the first 1000 groups of data from sample 1 and the first 900 groups of data from sample 2 are selected for the prediction. The results obtained are shown in Figures 11 and 12, respectively. They show that the prediction results' $95 \%$ confidence interval is too broad. Additionally, the error is deemed to be great. 


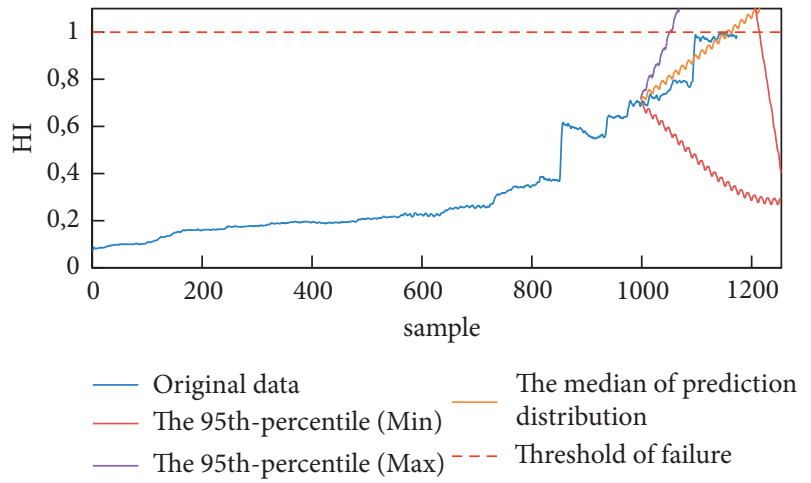

FIgURE 11: ARIMA-based prediction results for sample 1 (2).

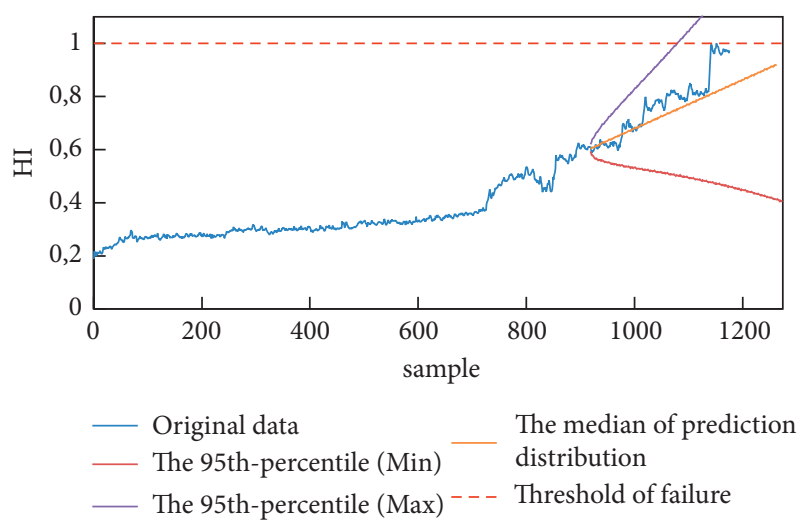

FIGURE 12: ARIMA-based prediction results for sample 2.

\section{Conclusions}

To evaluate and predict the performance status of renewable energy vehicle reducers in real time, this paper puts forward a data-driven method to predict the working life of a renewable energy vehicle reducer. An accelerated life test is conducted on a three-motor test bed, and life-cycle data are obtained from two renewable energy vehicle gearboxes to verify the proposed algorithm. The experimental results show that (1) the proposed method constructs a suitable trend index which is able to represent the current degradation condition and predict the future degradation trend of renewable energy vehicle reducer. (2) Relative to the traditional ARIMA model, the particle-based life prediction method receives better prediction results with a more convergent distribution. In future research, more lifecycle tests will be carried out on renewable energy vehicle gearboxes, and more experimental verifications will be conducted.

\section{Data Availability}

The processed data required to reproduce these findings cannot be shared at this time as the data also form part of an ongoing study.

\section{Conflicts of Interest}

The authors declare that they have no conflicts of interest.

\section{Acknowledgments}

This work was supported by the Fundamental Research Funds for Zhejiang Provincial Administration for Market Regulation (20190316) and the National Key Research and Development Program of China (2018Y FB2000502-2).

\section{References}

[1] Y. Qin, S. Xiang, Y. Chai, and H. Chen, "Macroscopic-microscopic attention in LSTM networks based on fusion features for gear remaining life prediction," IEEE Transactions on Industrial Electronics, vol. 67, no. 12, pp. 10865-10875, 2020.

[2] Y. Lei, N. Li, L. Guo, N. Li, T. Yan, and J. Lin, "Machinery health prognostics: a systematic review from data acquisition to RUL prediction," Mechanical Systems and Signal Processing, vol. 104, pp. 799-834, 2018.

[3] L. Guo, Y. Lei, N. Li, T. Yan, and N. Li, "Machinery health indicator construction based on convolutional neural networks considering trend burr," Neurocomputing, vol. 292, pp. 142-150, 2018.

[4] M. Rigamonti, P. Baraldi, E. Zio, I. Roychoudhury, K. Goebel, and S. Poll, "Ensemble of optimized echo state networks for remaining useful life prediction," Neurocomputing, vol. 281, pp. 121-138, 2018.

[5] B. Wang, Y. Lei, N. Li, and N. Li, "A hybrid prognostics approach for estimating remaining useful life of rolling element bearings," IEEE Transactions on Reliability, vol. 69, pp. 1-12, 2018.

[6] H. Liu, W. Song, Y. Niu, and E. Zio, "A generalized cauchy method for remaining useful life prediction of wind turbine gearboxes," Mechanical Systems and Signal Processing, vol. 153, Article ID 107471, 2021.

[7] A. K. Mahamad, S. Saon, and T. Hiyama, "Predicting remaining useful life of rotating machinery based artificial neural network," Computers \& Mathematics with Applications, vol. 60, no. 4, pp. 1078-1087, 2010.

[8] C. Cortes and V. Vapnik, "Support-vector networks," Machine Learning, vol. 20, no. 3, pp. 273-297, 1995.

[9] J. Deutsch and D. He, "Using deep learning-based approach to predict remaining useful life of rotating components," IEEE Transactions on Systems, Man, and Cybernetics: Systems, IEEE, vol. 48, no. 1, pp. 11-20, 2017.

[10] Z. Chen, X. Tu, Y. Hu, and F. Li, "Real-time bearing remaining useful life estimation based on the frozen convolutional and activated memory neural network," IEEE Access, vol. 7, pp. 96583-96593, 2019.

[11] Y. Pan, R. Hong, J. Chen, J. Singh, and X. Jia, "Performance degradation assessment of a wind turbine gearbox based on multi-sensor data fusion," Mechanism and Machine Theory, vol. 137, pp. 509-526, 2019.

[12] X. Chen, Z. Shen, Z. He, C. Sun, and Z. Liu, "Remaining life prognostics of rolling bearing based on relative features and multivariable support vector machine," Proceedings of the Institution of Mechanical Engineers-Part C: Journal of Mechanical Engineering Science, vol. 227, no. 12, pp. 2849-2860, 2013.

[13] B. Wang, Y. Lei, T. Yan, N. Li, and L. Guo, "Recurrent convolutional neural network: a new framework for remaining useful life prediction of machinery," Neurocomputing, vol. 379, pp. 117-129, 2020.

[14] L. Guo, N. Li, F. Jia, Y. Lei, and J. Lin, "A recurrent neural network based health indicator for remaining useful life 
prediction of bearings," Neurocomputing, vol. 240, no. 31, pp. 98-109, 2017.

[15] X.-S. Si, W. Wang, C.-H. Hu, and D.-H. Zhou, "Remaining useful life estimation-a review on the statistical data driven approaches," European Journal of Operational Research, vol. 213, no. 1, pp. 1-14, 2011.

[16] N. Li, Y. Lei, J. Lin, and S. X. Ding, “An improved exponential model for predicting remaining useful life of rolling element bearings," IEEE Transactions on Industrial Electronics, vol. 62, no. 12, pp. 7762-7773, 2015.

[17] X. Liu, Y. Jia, Z. He, and J. Zhou, "Application of EMD-WVD and particle filter for gearbox fault feature extraction and remaining useful life prediction," Journal of Vibroengineering, vol. 19, no. 3, pp. 1793-1808, 2017.

[18] H. Teng, J. Zhao, X. Jia, J. Yunxian, Z. Xinghui, and C. Liying, "Experimental study on gearbox prognosis using total life vibration analysis," in Proceedings of the 2011 Prognostics and System Health Managment Confernece, May-2011.

[19] X. Li, V. Makis, Z. Zhao, H. Zuo, C. Duan, and Y. Zhang, "Optimal Bayesian maintenance policy for a gearbox subject to two dependent failure modes," Quality and Reliability Engineering International, vol. 35, no. 2, pp. 659-676, 2019.

[20] L. Sun, Y. Jia, L. Cai, and X.-h. Zhang, "Residual useful life prediction of gearbox based on particle filtering parameter estimation method," Journal of Vibration and Shock, vol. 32, no. 6, pp. 6-12+23, 2013.

[21] F. Cheng, L. Qu, W. Qiao, and L. Hao, "Enhanced particle filtering for bearing remaining useful life prediction of wind turbine drivetrain gearboxes," IEEE Transactions on Industrial Electronics, vol. 66, no. 6, pp. 4738-4748, 2019.

[22] F. Yang, M. S. Habibullah, T. Zhang, Z. Xu, P. Lim, and S. Nadarajan, "Health index-based prognostics for remaining useful life predictions in electrical machines," IEEE Transactions on Industrial Electronics, vol. 63, no. 4, pp. 2633-2644, 2016.

[23] D. Wang, K.-L. Tsui, and Q. Miao, "Prognostics and health management: a review of vibration based bearing and gear health indicators," IEEE Access, vol. 6, pp. 665-676, 2018.

[24] F. Camci, K. Medjaher, N. Zerhouni, and P. Nectoux, "Feature evaluation for effective bearing prognostics," Quality and Reliability Engineering International, vol. 29, no. 4, pp. 477486, 2013.

[25] C. Li, X. Zhu, X. Li, Q. Zhu, and G. Yu, “The bearing health condition assessment method based on alpha-stable probability distribution model," in Proceedings of the 2016 Prognostics and System Health Management Conference (PHMChengdu, pp. 1-5, IEEE, Chengdu, China, 2016 October.

[26] M. Qiu, W. Li, F. Jiang, and Z. Zhu, "Remaining useful life estimation for rolling bearing with SIOS-based indicator and particle filtering," IEEE Access, vol. 6, pp. 24521-24532, 2018.

[27] X. Jin, J. Fan, and T. Chow, "Fault detection for rolling-element bearings using multivariate statistical process control methods," IEEE Transactions on Instrumentation \& Measurement, vol. 68, pp. 1-9, 2018.

[28] B. P. Duong and J.-M. Kim, "Non-mutually exclusive deep neural network classifier for combined modes of bearing fault diagnosis," Sensors, vol. 18, no. 4, Article ID 1129, 2018. 